\title{
Japanese Patients' Preferences for Receiving Cancer Test Results While in the United States: Introducing an Advance Directive for Cancer Disclosure
}

\author{
MICHAEL D. FETTERS, M.D., M.P.H., M.A. ${ }^{1}$ and YUICHIRO MASUDA, M.D. ${ }^{2}$
}

\begin{abstract}
Background: While disclosure of cancer is routine in the United States, it is not in Japan. The primary goals of this investigation were to describe overseas Japanese patients' preferences for participation in decisions about cancer; to delineate patients' beliefs about how physicians should make cancer disclosure decisions; and to assess patients' attitudes about a advance directive for cancer disclosure.

Methods: This ethnography utilized data from participant observations, 30 interviews, and lay materials. Using the analytic technique of immersion and crystallization the data were organized around major themes and subthemes.

Results: The mean age of the 30 participants was 38 years and most $(n=20)$ were female. While most patients expressed preferences to be told the results of cancer testing, the number who did not want to be told increased with more advanced cancer stage. Many participants believed decisions about disclosure should be influenced by a patient's personality, age, or gender, although the relative importance of these factors was variable. Virtually all believed circumstances existed when fully competent patients should not be told the cancer diagnosis. Most participants were supportive of using an advance directive for cancer disclosure.

Discussion: These participants' beliefs about many aspects of the management of cancer test results differ from recommended approaches to conveying cancer test results and discussing the cancer diagnosis in the United States. Most encourage the use of an advance directive for cancer disclosure as mechanism for physicians to elicit patients' unique preferences for participation in decision making about cancer.
\end{abstract}

Doctor: You have an ulcer. You have to have surgery or you will die. We may have to take out half of your stomach. It has to be cut out.

Patient: I can't! We are in the middle of filming. Can't it wait?

Doctor: This is your life.

Patient: You have to promise. Take responsibility. (Doctor looks shocked)

Doctor. Without a trusting relationship, I can't take care of you. I'll introduce you to another doctor.

Patient: Okay doctor, I will have surgery.

(Later with patient's wife, Mariko.)

1Japanese Family Health Program, Department of Family Health Systems, Ann Arbor, Michigan.

${ }^{2}$ Department of Geriatric Medicine, Nagoya University School of Medicine, Nagoya, Japan. 
Doctor: He has cancer. (Mariko has panicked look on face. She begins coughing, choking, and gasping. She vomits into the sink. The doctor holds her shoulders from behind).

Are you Okay?

Mariko: He doesn't know?

Doctor: I have told him it is an ulcer.

Mariko: Absolutely don't tell. He couldn't take it.

From the movie Daibyounin (The really sick person)

There are people so addicted to exaggerating they can't tell the truth without lying.

From a Chinese fortune cookie

\section{INTRODUCTION}

A N IMPORTANT CHALLENGE in medical care occurs when patients' and their families' cultural beliefs about best health care practices differ from the procedures their physicians are accustomed to following. ${ }^{1-8}$ These differences may be particularly relevant when cancer is diagnosed, an emotionally trying time for patient, family, and physician alike. They can be especially profound when patients and their physicians have different cultural backgrounds. ${ }^{9-11}$

Different approaches to disclosure of a cancer diagnosis illustrate this tension. In most countries of the world, physicians do not routinely disclose a diagnosis of cancer to their patients. ${ }^{12-18}$ As in many of these countries, Japanese physicians typically have been taught to avoid candidly disclosing bad news such as a cancer diagnosis to the patient. ${ }^{19}$ Recently, the merits and approaches to nondisclosure and disclosure of cancer in Japan have generated significant interest among the general population, physicians, medical ethicists, and the government. ${ }^{20-36}$

When Japanese people come to the United States, they move from a country where nondisclosure predominates to one where disclosure is nearly ubiquitous. As in other cultural groups in the U.S., some Japanese are here by choice with a desire to be integrated into U.S. society. Others such as political refugees or those who are transferred on temporary assignment by their companies, do not necessarily come by choice, nor are they necessarily interested in adopting U.S. customs. In the global economy, the number of people on temporary work assignments abroad such as Japanese employees and their families will continue to grow. In such circumstances when cultural norms about cancer disclosure conflict, the issue of how to communicate with patients about cancer becomes particularly pertinent.
Advance directives are advocated as a means of extending patient autonomy to circumstances when the individual is no longer competent to designate his/her preferences for use of life-sustaining treatment and other medical therapies. ${ }^{37-39}$ Given the diversity in cancer disclosure practices and patient preferences to know if affected with cancer, Asai and others ${ }^{40,41}$ have proposed and implemented in Japan an advance directive for cancer disclosure as a proactive means to guide a physician's cancer disclosure practices for individual patients. In his survey of new patients at an outpatient internal medicine clinic, $76 \%(n=228)$ thought that physicians should routinely ask patients if they would want to know about a cancer diagnosis. ${ }^{41}$ While Freedman ${ }^{42}$ has suggested the approach of "offering truth" after cancer is diagnosed, to our knowledge no comparable advance directive documents or techniques have been used in the United States for determining patient preferences for disclosure prior to establishing the cancer diagnosis.

The purposes of this research were: (1) to describe overseas Japanese patients' preferences for communication and participation in medical decisions about cancer; (2) to delineate patients' beliefs about how physicians should make decisions about whether and how to disclose a cancer diagnosis to a patient; and (3) to assess patients' attitudes about the use of an advanced directive for cancer disclosure.

\section{METHODS}

We conducted an ethnography of overseas Japanese patients presenting for outpatient care to the Japanese Family Health Program, an initiative designed to provide linguistically and culturally sensitive care to the population of Japan- 
ese people residing in Southeastern Michigan. The project was approved by the University of Michigan Health Systems Institutional Review Board. Through the process of reflexivity, we reviewed our personal and professional backgrounds, and through this process sought to identify our own biases. ${ }^{43-45}$

Eligibility criteria required the participant to be an overseas Japanese patient who presented during the study period for a comprehensive health maintenance examination (ningen dokku), or a patient who needed diagnostic testing to rule out an illness such as lung, breast, cervical, stomach, or colon cancer. The participants were interviewed at the time one or more cancer test(s) was (were) ordered in order to minimize the artificiality and hypothetical nature of the inquiry.

Patient recruitment occurred in a community setting from 1 July 1995 until 31 December 1996. Fourteen months into the study, the clinical program moved to a new site where data collection continued an additional four months. Patients were intentionally selected to obtain maximal variation in patient gender and age distribution. ${ }^{46}$

The semistructured instrument was designed to determine patients' preferences for receiving the results of cancer screening or diagnostic tests (see Appendix). After obtaining informed consent for participation, patients were asked a series of open-ended questions about their preferences for receiving the results of cancer tests. The major variables of interest included: the mechanisms by which patients preferred to receive information; the influence of cancer stage, cancer type, patient age, patient gender, and strong or weak patient personality on whether to disclose a cancer diagnosis; and personal, family, or acquaintance experiences with cancer. By design, new questions were added in the course of the investigation in order to explore new issues raised by the participants' responses. Examples of added questions included inquiry about preferences for disclosure and patient beliefs about how age, gender, cancer stage, and patient personality should influence disclosure of information to the patient.

The tapes were transcribed verbatim and the transcripts served as the primary data source. ${ }^{47}$ Field jottings and observations were used to provide context for the audiotapes. ${ }^{48}$ The interviews were conducted after completing the patient's clinical examination. Their preferences were recorded in the chart and the results were subse- quently conveyed according to their wishes. The criterion of theoretical saturation was used as the primary endpoint of data collection. 49,50

The data were analyzed using the process of immersion/crystallization. ${ }^{43,51}$ We "immersed" ourselves in the data by reviewing the transcripts and other field notes multiple times until the major themes "crystallized" out. We then went back to the text to assess the accuracy of the themes and sub-themes. We synthesized from the data a cohesive overview of the content and used illustrative quotes and metaphors generated by the participants. We tabulated the responses to the questions asked of most or all the participants. Because new questions were added in the course of data collection, not all questions were asked of all participants.

\section{RESULTS}

\section{Demographics}

Forty-three people met eligibility criteria. Of these, 4 returned to Japan before they could be interviewed, and 9 were willing to participate, but could not complete the interview for feasibility issues; 10 nonparticipants were female, and 3 were males. All of the remaining 30 individuals agreed to participate (Table 1). Of these, 20 $(67 \%)$ were females. The number of children ranged from none to 3 . Most participants had lived in the United States for at least a year, with a minority having lived in other foreign countries. Most participants had personal experiences with family or friends affected with cancer (Table 2). When cancer had been disclosed to afflicted significant others, all expected positive outcomes; non-disclosure sometimes yielded negative outcomes (Fig. 1).

Patient responses during the investigation fall into four major categories: communicating to patients about cancer tests; patients' advice to physicians for deciding to disclose cancer; patients' personal experiences with cancer; and patients' beliefs and attitudes about the advance directive for cancer disclosure (Table 3 ).

\section{Communicating cancer test results}

Preferred methods to receive results. Table 4 summarizes patient's preferences for receiving results (letter, telephone, or in person), according to the result of the screening or diagnostic test; that is, 
TABle 1. Demographics

\begin{tabular}{lc}
\hline & $\mathrm{n}=30$ \\
\hline Female & 20 \\
Male & 10 \\
& \\
Occupation & 18 \\
$\quad$ Housewife/not working & 4 \\
Engineer & 4 \\
Manager & 3 \\
Company worker & 1 \\
Nurse & \\
& \\
Home in Japan & 14 \\
Aichi prefecture & 4 \\
Tokyo & 3 \\
Kanagawa prefecture & 2 \\
Chiba prefecture & 2 \\
Hiroshima prefecture & 5 \\
Other & \\
Number of children (mean) & 1.5 \\
Months in United States (mean) & 31 \\
Months lived in other foreign country & 0.5 \\
(mean) & \\
Months planned to live in US (mean) & 56 \\
Age (mean) & 38 \\
Years of education (mean) & 15 \\
\hline
\end{tabular}

ancludes Saga, Fukuoka, Okayama, Tochigi, Osaka.

normal, early stage ( $80 \%-90 \%$ survival rate), medium stage $(30 \%-70 \%$ survival rate), or advanced stage ( $<20 \%$ survival rate). For normal results, most patients preferred disclosure by letter $(50 \%)$ or in person $(37 \%)$. Surprisingly, some participants voiced aversion to receiving results over the phone since they would not have a tangible record of their results; for fear of not understanding the content; and because using the phone felt informal. For early, medium, and advanced stage cancers, the percent of patients who preferred to be told in person was $93 \%, 70 \%$, and $60 \%$ respectively.

Patient preferences for disclosure of cancer results. Most patients wanted to be told if cancer was diagnosed, but their desire for disclosure decreased as the cancer stage became more advanced (Fig. 2). While $100 \%$ wanted to know if results were normal, this number decreased to $90 \%$ of participants if early stage, $70 \%$ of participants if medium stage, and $60 \%$ of participants if advanced stage.

Two reasons were commonly given for being told. First, many patients feel the need to make life plans. A second reason is to learn the details and implications of the illness. Those who did not desire disclosure at the medium and advanced stage fell into three areas: (1) wanted a family member to be told, (2) simply did not want to be told, and (3) couldn't answer the question.

Family member involvement. Most participants wanted family members involved when receiving results positive for cancer (Table 5). In this circumstance, most of the male and female participants indicated their preference to receive notification of the need to schedule an appointment to discuss an abnormal result by mail or phone. They indicated they would come with their spouse to the appointment and learn the news together.

Exceptions occurred mostly among individuals who indicated their preference for disclosure of the diagnosis by stage, especially, for an advanced stage cancer. There were other exceptions as well. One male patient asked that his wife not be present at the time of disclosure as he preferred to disclose this information to her. A minority of the participating men stated that if they had cancer that they would want to be told, but they would not want their wives told if their wives had cancer.

There were also a minority of women who stated that they would want to be told if cancer was diagnosed in themselves, but that they would not want their husbands to be told if cancer was diagnosed. There was one couple for whom the wife indicated her preference to be told

Table 2. Participants' Personal Experiences WITH FAMILY OR FriendS AfFlicted WITH CANCER

\begin{tabular}{lr}
\hline & $\mathbf{n}$ \\
\hline $\begin{array}{lr}\text { Personal experience with family or friend } \\
\text { having cancer }\end{array}$ & 27 \\
Relationship to person & \\
Grandparent & 23 \\
Parent & 11 \\
Aunt or uncle & 13 \\
Other relative & 6 \\
Friend & 1 \\
Acquaintance & 2 \\
Cancer disclosed to the person & \\
Cancer disclosed & 12 \\
Cancer not disclosed & 42 \\
Uncertain & 2 \\
\hline
\end{tabular}

aColumn does not add to 27 because multiple responses were possible. 
Table 3. Major Themes and Subthemes

Communication and disclosure
Conveying cancer test results
Patient preferences for cancer disclosure
Involving family members
Patients' advice for cancer disclosure decision making
Patient's personality
Age
Gender
Prognosis
Cancer type
Personal experiences
Cancer in the family
Cancer in friends
Advanced directives for disclosure
Acceptability of advanced discussions

regardless of the circumstances, and in a separate interview, her husband was adamantly opposed to disclosure of a cancer diagnosis to her.

\section{Factors influencing cancer disclosure decision making}

Virtually all participants indicated that in some circumstances it would be preferable for physicians to not disclose a cancer diagnosis to a patient. The reasons for not disclosing the information varied from person to person. No specific factors, such as age, gender, personality, and cancer stage, were agreed on to use in making a decision to disclose, or not disclose, the cancer diagnosis.

Reasons for nondisclosure or disclosure of cancer diagnosis. Among the varied reasons for withholding the cancer diagnosis was the use of deception with intent of motivating the patient to be cured.
Some participants referred to the "mental" side of curing cancer and encouraged physicians to avoid causing psychological stress.

Participants also gave a series of reasons for disclosing cancer to the patient. One opinion was simply the aversion to not knowing. A male participant said, "I think for myself that knowing everything, the truth, is the best ... Just the thought of thinking, 'I bet it is, I bet it is,' and then not being told anything is scary." Other less frequently raised factors included patients having particular religious beliefs and patients having a good understanding of science and, therefore, an ability to think objectively about the problem and its management.

Impact of specific factors on decision to disclose. The participants who were asked what advice they would give, were all questioned about five factors (personality, age, gender, prognosis, and cancer type) that could influence the physician's decision to disclose the diagnosis.

Personality. Patient's personality had the most consistent support for use as a factor in determining whether to disclose the cancer diagnosis. That is, someone who has a "weak" personality should not be told. One woman explained, "For someone who worries too much, paradoxically, they become obsessed with only the illness, and it becomes difficult to treat the person ... There was a man in my husband's company whose health was deteriorating, and he was worried that it was cancer. He couldn't play golf, and he couldn't bring food to his lips. I really think it is better not to tell someone like that."

When asked for advice on how to make a determination of who had a "weak" personality, some laughed as though that was something

Table 4. Patient Preferences for Receiving Cancer Test Results

\begin{tabular}{lcccc}
\hline $\mathrm{n}=30$ & $\begin{array}{c}\text { Normal } \\
\text { result }\end{array}$ & $\begin{array}{c}\text { Early } \\
\text { stage } \\
\text { cancer }\end{array}$ & $\begin{array}{c}\text { Medium } \\
\text { stage } \\
\text { cancer }\end{array}$ & $\begin{array}{c}\text { Advanced } \\
\text { stage } \\
\text { cancer }\end{array}$ \\
\hline Telephone & 1 & 0 & 0 & 0 \\
Letter & 15 & 1 & 0 & 0 \\
In person & 11 & 26 & 20 & 17 \\
Any of these & 3 & 1 & 1 & 1 \\
Tell family member & 0 & 0 & 2 & 4 \\
Do not disclose to me & 0 & 1 & 1 & 4 \\
Could not answer & 0 & 1 & 4 & 3 \\
Not asked & 0 & 0 & 2 & 1 \\
\hline
\end{tabular}




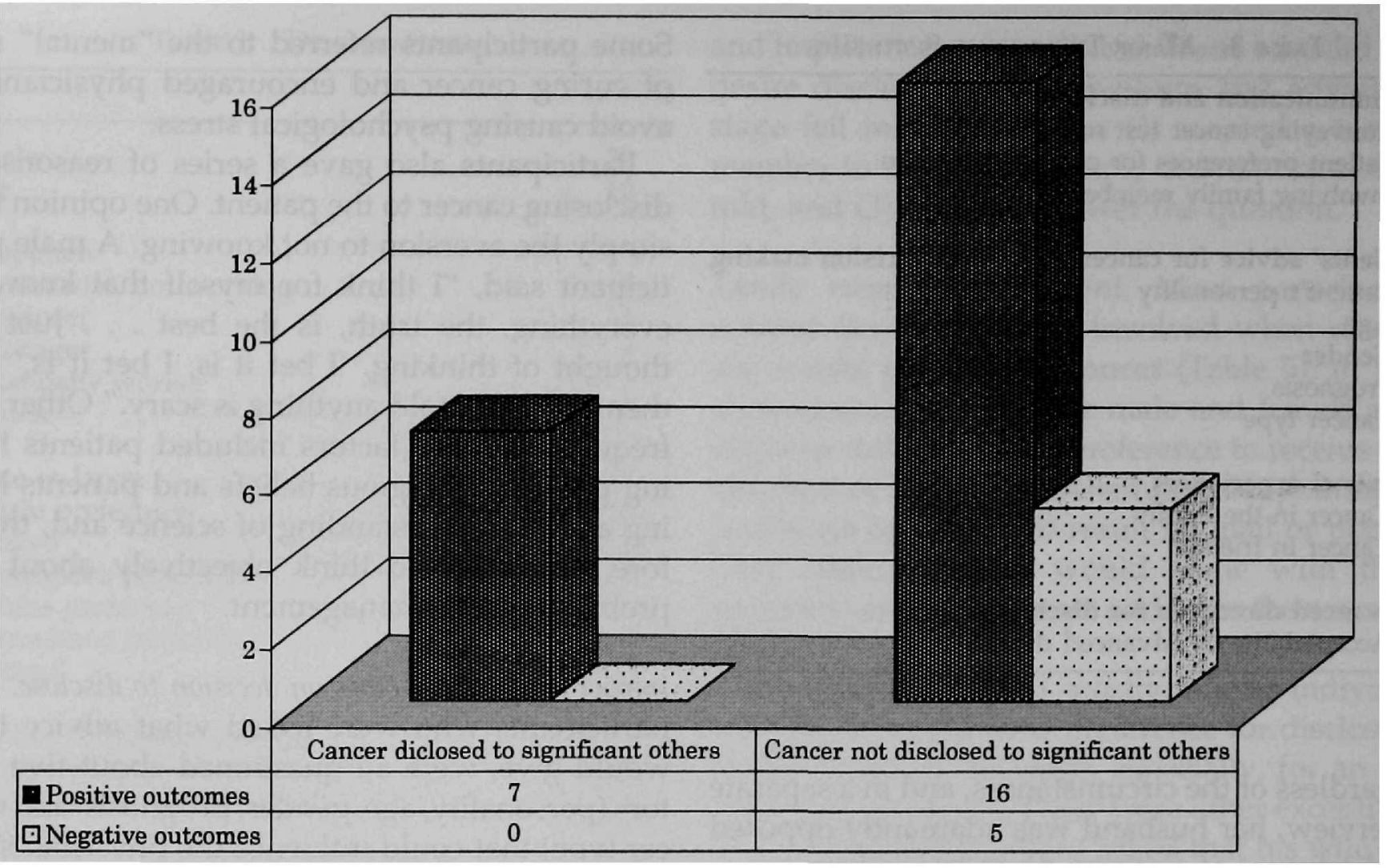

FIG. 1. History of cancer disclosure to significant others.

physicians were supposed to already know, while others held their breath and advised it was difficult to do. The most common advice was to talk to family members in order to get an assessment of the person's personality. One respondent illustrated, "I think personality is important. Since you won't know unless you're watching the person all the time, I can't help but feel that the family knows best."

The circumstance of the patient having a strong personality was also addressed. One women said, "I think it is better to tell if it someone who is mentally strong and tough, who will take in the news for what it is, and make the best of it." The problem of relying on the patient's personality was also noted by a minority. One illustrative response by a man was, "I have my work face, and my home face, and there is no one who knows both of my selves. It is difficult to judge who knows my personality."

Age. Age elicited strong support as a criterion, but there were conflicting age cutoffs. Some felt that older people should not be told; others thought the elderly were more likely to accept the diagnosis well and should be told. For example, one male explained,
I think younger people are shocked more than older people. I think that someone about the age of 90 recognizes that whether or not afflicted with cancer, that their time will come. Also, for someone who is 50 or 60 years of age, they can still work a lot and enjoy life, so I think telling them will allow them to enjoy the remainder of their lives.

Several of those supporting telling younger people were mothers with small children, who wanted to be able to plan for the care of their children after mother's death from cancer.

Gender. While most respondents felt gender was not important, six participants voiced opinions about the role of gender. These six responses indicated three conflicting rationales. First, some (male and female) participants indicated a belief that men should be told because they had important social responsibilities. Second, there was a belief that women should be told ambiguously because they overreact and think about too many contingencies. A third rationale was that men, in general, were less able to handle bad news than women were and, thus, it would be easier to tell women than men. 
Table 5. Patient Preferences for Method and Timang of Disclosure of Cancer to Family Members

\begin{tabular}{lr}
\hline & $n=30$ \\
\hline $\begin{array}{l}\text { Preferred family member for disclosure } \\
\text { Spouse }\end{array}$ & 25 \\
Not asked & 5 \\
& \\
Method of disclosure to family member & 22 \\
Be told by physician & 4 \\
Be told by the patient & 1 \\
Be told by the patient and then the & \\
physician & 3 \\
Preference depends on prognosis & \\
& \\
Timing of disclosure & 3 \\
Disclosure before telling the patient & 10 \\
Disclosure when telling the patient & 9 \\
Disclosure after telling the patient & 6 \\
Preference depends on prognosis & 2 \\
Not asked &
\end{tabular}

Prognosis. As indicated above in patient preferences for conveying results, a worse prognosis is associated with a greater likelihood that the patient will not want the cancer diagnosis revealed (Fig. 2). A number of participants reflected particularly about the circumstance of a terminal cancer. For example, one explained,

If the patient is told 'terminal cancer' he or she wonders about death, and it just gets translated into untreatable ... (pause) There is a fear of death, isn't there? I think the problem is how to soften that fear of death. If you don't do a good job softening the

Table 6. Attitudes About Advance Directives for Cancer Disclosure

$n=30$

Does asking about your preference for

cancer disclosure make you uncomfortable?

Yes

No

Unsure

Not asked

Attitudes about discussing patient

preferences for advanced directives for

cancer disclosure

Strongly support

Somewhat support

Strongly opposed

Somewhat opposed

Could not answer

Unclear truth, because for someone with cure rate of $10 \%$ or less, someone with untreatable disease, I think just disclosing cancer is irresponsible . . . (pause) While I think there is movement in the direction of disclosure in Japan, I think this is largely a discussion of people with curable disease.

Patients who preferred disclosure varied with the stage of the cancer. The first subgroup, is male patients who prefer not to know if there is less than a $50 \%$ chance of cure. The second sub-group, female and male patients, prefer not to be told if there is terminal cancer.

Cancer type. Most patients stated that the cancer type (breast versus stomach versus colon, etc.) should not influence whether the diagnosis is disclosed. Of those who thought it should, they qualified their response that some cancers were more likely to be diagnosed in an early stage. Thus, considering stage, patients overwhelmingly felt that cancer type should not influence whether the patient should be told.

Participants' advice on deciding what to say. Participants were asked to give advice on how doctors should decide the difficult issue of who ought to be told a cancer diagnosis. This generated a variety of responses. Among these was advice for using deception with intent of motivating the patient to be cured. One participant said,

In order to have the best chance for cure, I think agreement can be elicited by using an eloquent expression. If you start treatment saying, "Even by starting treatment now, at best there is a $10 \%$ chance of cure," or you start treatment saying, "If you try really hard, I think there a $50 \%$ chance of cure." I think there is a very different outcome. It is OK to tell a lie if you have a good outcome.

These participants referred to the "mental side" of curing cancer and encourage physicians to avoid causing psychological stress. For example, they indicated that part of doctoring is giving the patient hope. This sentiment was expressed by one woman who advised,

In the case of a cancer that is $100 \%$ fatal, I don't want you to say, "There is $100 \%$ chance it is fatal." Even if you think it is 


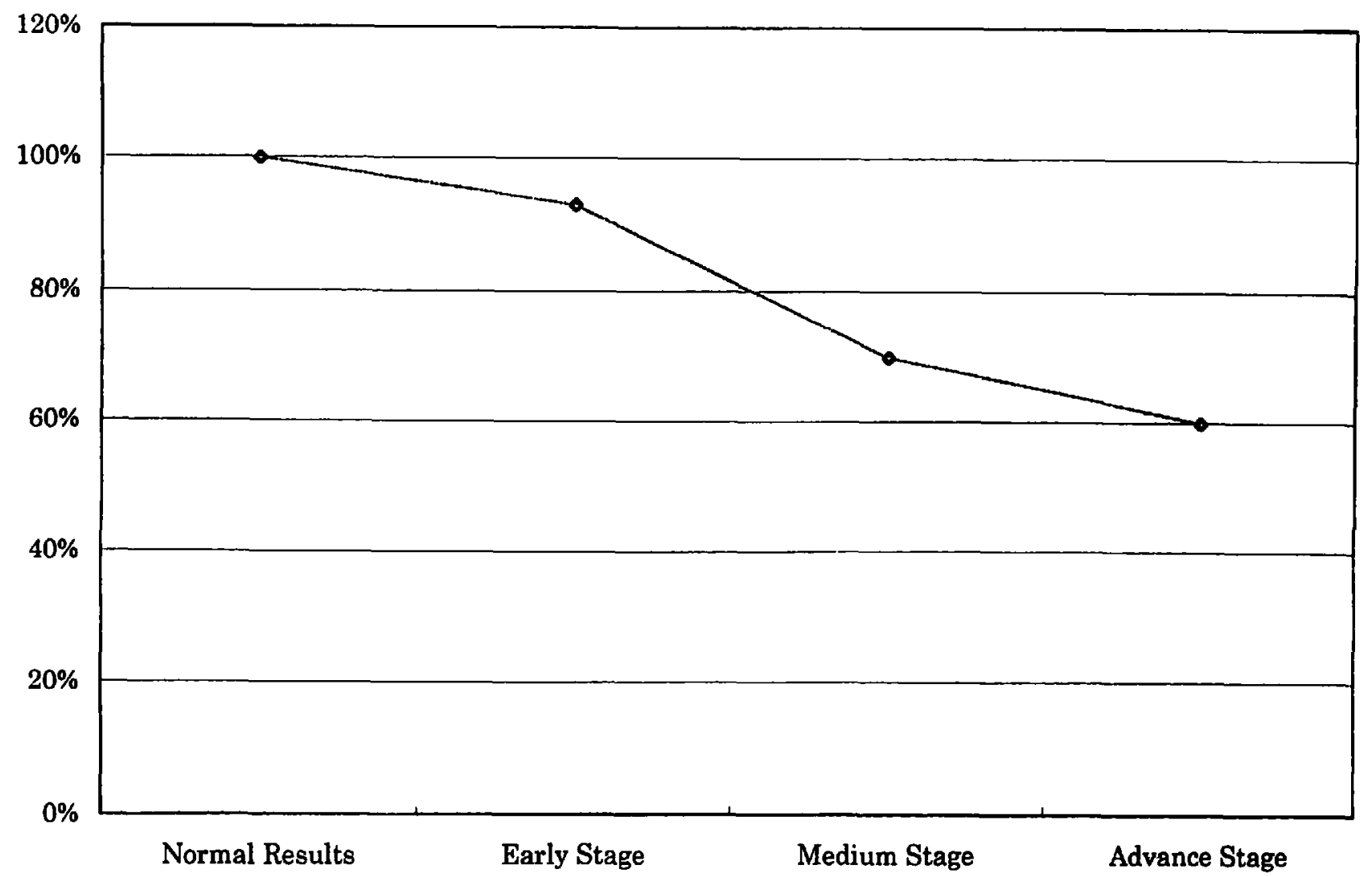

FIG. 2. Percentage of patients requesting disclosure by cancer stage.

hopeless, I hope you will speak in a way that gives at least a little hope.

\section{Acceptability of advance directives for cancer disclosure}

Patients were asked whether they believed the use of a questionnaire and advanced discussion was an acceptable way of approaching patients who have a screening or diagnostic test for a possible cancer diagnosis (Table 6). This line of questioning generated four responses.

The vast majority of participants voiced enthusiastic support for advance directive use. One woman posited, "When asked my preference for disclosure while healthy, as you are doing, an honest preference comes out. So I think asking is best. When asked, 'Do you want to know? Do you not want to know' I think everyone will ask you to do it." A male participant echoed her thoughts and said, "I think it is really important to conduct a survey and ask for people's opinions about whether they want to be told they have cancer. I think especially in Japan that things are changing. There are people with the same opinion as me, and people with different opinions, so I hope you will refer to this and use it in your treatment."

The other three responses were more cautious, and one was even very negative. Some were con- cerned that raising the issue caused uneasiness, especially for someone who presented with symptoms. One woman explained, "If you spring this discussion on me when I am ill, it makes me wonder whether or not I have cancer." Another concern with an advanced directive for disclosure was impact on trusting the doctor. One man explained, "If you start out by saying that your preference is not to be told, the trustworthiness of the discussion thereafter drops." The third response reflected resentment that doctors would even consider not disclosing the truth. One man expressed his concern that this approach was a waste of time. He shared his belief that patients ought to be told the truth about their health regardless of the problem. He believed there was an inordinate obsession with cancer and a lack of adequate discussion in general about illness.

\section{DISCUSSION}

Communication and participation in

medical decision about cancer

These participants' beliefs regarding many aspects of communication about cancer test results differ from standard approaches in the United States. No participant consensus on one best way 
to convey results to patients was identified, but the vast majority of participants preferred to get normal results in writing rather than personally from the physician in the office or by telephone. This contrasts with similar research done in the United States by Lind et al. ${ }^{52}$ who found that female patients preferred getting normal mammogram results by phone call. Still, most patients with an abnormality in both our study and the Lind study preferred to learn of an abnormal result in person.

Most patients expressed a preference to be told the details of their illness if an early stage cancer was diagnosed. The number with a clear preference for disclosure declined as the prognosis worsened, a trend supported by research conducted in Japan. ${ }^{41,53}$ These beliefs also parallel the trend for Japanese physicians to disclose less information about cancer as the prognosis worsens. ${ }^{30,54}$

The patients who did not want to be told if cancer was diagnosed consistently identified their spouses as the individuals to whom they wished the information to be conveyed. All participants were married and in their third to fifth decades of life. Because it is uncommon for an older parent or other family members besides children to live with expatriate families in the United States, for most participants the only plausible substitute decision-maker would have been the spouse. It is conceivable that their preferences might differ in Japan, though previous research has identified similar patterns of decision making by family, especially the spouse, in Japan. ${ }^{19,30,31}$

\section{Patient beliefs about how to disclose cancer}

These participants' responses do not consistently support respect for patient autonomy and cancer disclosure. Regardless of their own preferences, most participants believe that under certain circumstances some patients, including those who are competent, should not be told a cancer diagnosis. This implies they believe there should be flexibility for cancer disclosure decision making, because some prefer disclosure, others do not, and no predictors are known of which people would fit into each pattern.

Figure 3 graphically summarizes patients' advice to weigh a variety of factors when deciding whether to disclose cancer. While most agreed a series of factors should be weighed in decision making about cancer disclosure, and cumula- tively these ought to determine whether the patient should be told, no consistent agreement was found about what factors are most important and how those factors should influence decisions. These participants believe that the cancer patient's personality, or ability to handle bad news, is a crucial consideration, though they also indicate other factors such as age, gender, etc., to be relevant as well. Most believe that the spouse is the best judge of the patient's ability to handle this information. Interestingly, physicians have identified the same factors as relevant for deliberation about cancer disclosure and end-of-life decision making. ${ }^{30,55}$

Many have criticized Japanese physicians' neglect to disclose cancer and, hence, for not practicing informed consent and respecting patient autonomy. However, these data suggest that Japanese physicians and patients share similar thinking about cancer disclosure. Concordant views include, first, the belief that some people exist to whom a cancer diagnosis should not be disclosed and, second, the belief that contextual issues such as the patient's personality, age, gender, cancer stage, and other factors are crucial in decisions about whether to disclose a cancer diagnosis. This suggests that Japanese physicians' behaviors are based in extant, social norms and not just in the cultural norms of Japanese medicine.

\section{Attitudes about an advanced directive for cancer disclosure}

As illustrated in the movie Daibyounin, physician and family decision makers are in a quandary if the patient's preferences are unknown. Once the patient is told, no retreat may be made. This very complex circumstance is in dire need of simplification.

Figure 3 illustrates how the advance directive for disclosure could circumvent the complex system of decision making about disclosure. At time $t_{0}$, the patient can direct disclosure to others such as the family or oneself. After cancer is diagnosed at time $t_{1}$, the information is disclosed at time $t_{2}$ to the person or others as directed by the patient. In the event the patient prefers not to be told, or does not want to make an advance directive for disclosure, decision making proceeds in the usual, complicated fashion with the physician and family weighing a series of factors $a, b, c \ldots$ $n$, as necessary for determining sufficient reasons 


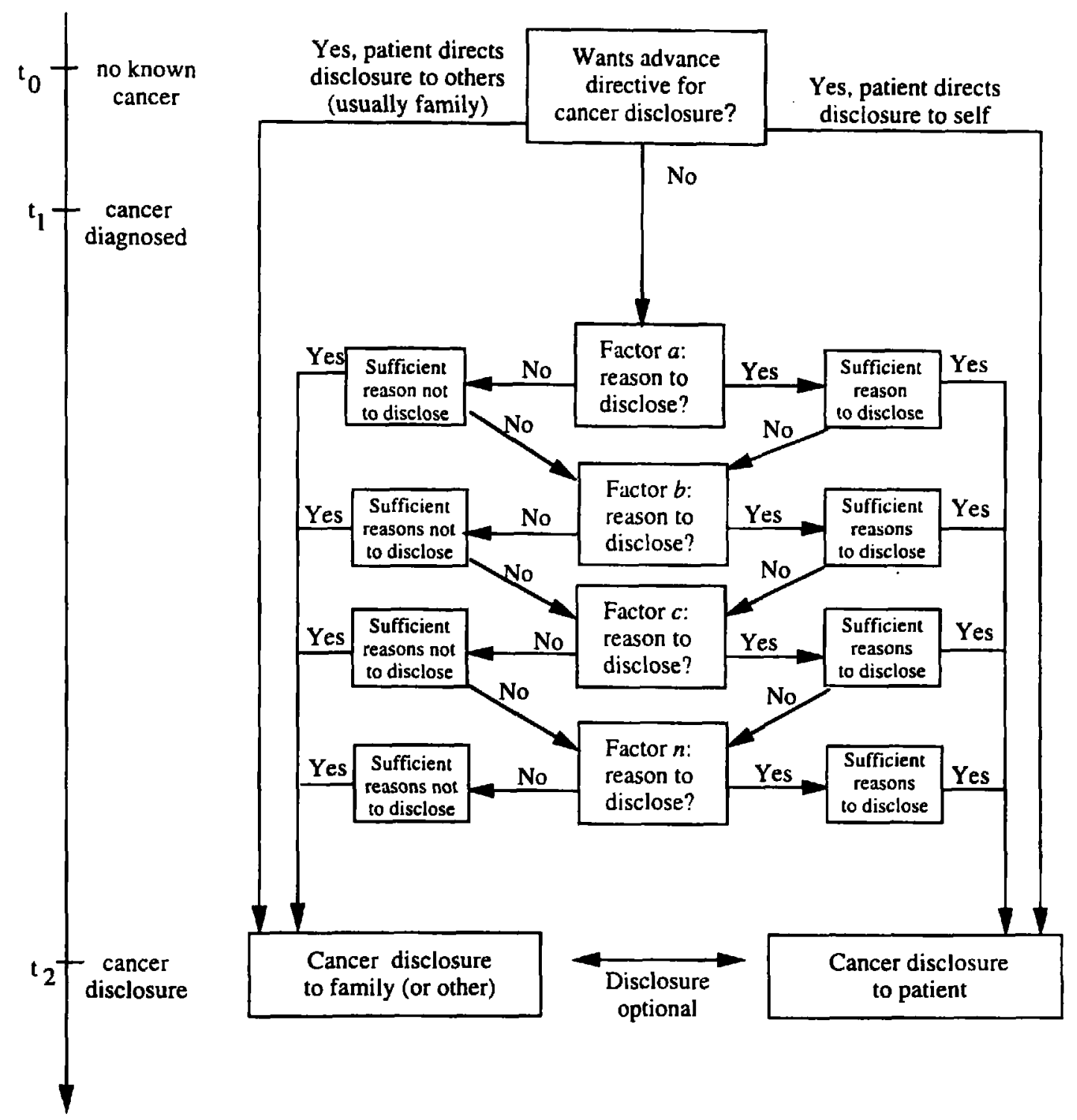

FIG. 3. Advance directives for cancer disclosure and cancer disclosure decision making.

to disclose or not disclose the information to the patient.

Most participants support asking patients in advance their preferences for disclosure when a screening or diagnostic test for cancer is ordered. Both personal discussion with the physician or in writing through a written document was acceptable. Most patients did not feel that asking them for their preferences made them uncomfortable. Rather, it was reassuring that they would be given information in a manner driven by their own preferences.

Since the implementation of advance directives for cancer disclosure in the Japanese Family Health Program, two Japanese patients have been diagnosed with cancer. Through an oral advance directive, both indicated an a priori preference to know all the details of their care. Among those patients indicating a preference to not be told a cancer diagnosis, none have been identified with cancer.
When a newly identified cancer patient asks in advance for non-disclosure, Western bioethics and Japanese values will clash. If the U.S. clinician-investigator (M.F.) is to live up to the patient's advance directive, he will have to deceive the patient. Steeped in a tradition of informed decision making and cancer disclosure, the mere thought is discomforting, but provides an inkling of how uncomfortable Japanese physicians might feel about disclosing cancer. U.S. clinicians have not been trained to deceive the patient. Japanese physicians, who have been trained to protect patients by deceiving them, must feel similarly uncomfortable about disclosure.

The advance directive for cancer disclosure may provide a practical mechanism for communicating with patients with cancer. Unfortunately, the standard advance directive is far from foolproof. In the SUPPORT study, patient advance directives were not found to have a clinically relevant impact. ${ }^{56}$ Furthermore, while the 
number of people who have obtained directives is growing, most Americans do not have one and their physicians often don't even know the patient has completed one. ${ }^{57-61}$

There may be other pitfalls of using an advance directive for cancer disclosure as well. An oral advance directive, in particular, can most easily be respected if the patient is seen exclusively by the physician with whom it was discussed. The requirement to obtain informed consent before providing chemotherapy protocols, radiation treatments, and surgery has been codified in law. What are the implications for following the patient's preferences when it conflicts with the law?

Finally, the current data are not necessarily generalizable to all overseas Japanese people. Most of the participants were of child bearing or child rearing age. The paucity of retired individuals in the overseas population of the region precluded their enrollment in the study. Furthermore, most participants have financial security and health insurance, although these attributes are not unusual for the Japanese population, which has universal healthcare coverage and a low unemployment rate.

\section{CONCLUSIONS}

While some Japanese patients supported decision making reflecting patient autonomy and cancer disclosure for themselves or others, many simultaneously articulated beliefs that decisions should be situational and not driven by a hard and fast rule of cancer disclosure. Most felt there were circumstances when cancer should not be disclosed. While there was not absolute agreement on the specific factors to consider nor agreement on how to weigh them, they do articulate a multifactorial process of decision making that is consistent with processes identified in data collected from Japanese physicians. Thus, while a Japanese physician approach of nondisclosure of cancer conflicts with Western bioethics notion of patient autonomy and informed consent, there is patient support for these actions some of the time, but not all of the time. Physicians need to be wary that they are not so addicted to deceiving that they can never tell the truth. An advance directive for cancer disclosure offers a potential means for guiding which approach to disclosure should be followed.

The notion of an advance directive for cancer disclosure may have importance beyond this spe- cific cultural group. Despite broad advocacy by medical ethicists for cancer disclosure in the United States, subgroups of patients in the United States are opposed to being told. Blackhall et al. ${ }^{62}$ examined differences in preference for disclosure among $(n=200)$ patients 65 years of age and older who were living in Southern California. The participants who favored cancer disclosure varied significantly: African American (88\%), European American (87\%), Mexican American (65\%), and Korean American (47\%). Those favoring disclosure of a terminal prognosis were even fewer, African American (63\%), European American (69\%), Mexican American (48\%), and Korean American (35\%). Thus, even in the United States where disclosure is a near-ubiquitous approach, patient preferences for disclosure and participation in decision making vary. ${ }^{63}$ The lack of consistent beliefs among patients about the appropriateness of disclosure, and the consistently identified trend in this and other studies for more patients preferring not to be told the cancer diagnosis as the prognosis grows worse may raise particular problems for palliative care efforts.

To innovate is to renew, alter, or introduce new methods or devices. Use of advance directives in the United States have previously been reserved exclusively for patients who are incompetent to make decisions, as it is assumed that all competent patients either want to or should be informed and participate in their medical decisions. ${ }^{64,65}$ Medical ethicists do not agree on whether a culturally-based preference not to know information and not to participate in decision making should trump the hard-earned, but now routine, right to be informed and participate in decisions. Until consensus is reached, an advance directive for cancer disclosure offers clinicians a modest, innovative tool to facilitate patient participation in decision making.

\section{APPENDIX}

\section{Patient Questionnaire}

During your health check today, we will order one or more cancer diagnostic or screening test(s). The purpose of a screening test is to identify disease early when it is curable. Most cancer screening tests are negative. Still, screening tests can find both early stage, middle stage and advanced stage cancers. Diagnostic tests are done to look for disease when the patient has symptoms. 
As you may know, doctors in Japan often do not tell patients when cancer is found. But in the United States, doctors almost always tell patients the truth. Given this cultural difference, we would like to learn of your preference in advance of finding the results so we can follow your preference.

You are receiving tests for

1. Most cancer screening tests are negative for cancer. If your test result shows there is no cancer, how would you prefer to receive today's results?

2. How would you prefer to be told if it was an early cancer with an $80 \%-90 \%$ chance of cure?

3. How would you prefer to be told if it was a middle stage cancer with a $30 \%$ to $70 \%$ chance of cure?

4. How would you prefer to be told if it was an advanced stage cancer with $20 \%$ or less chance of a cure?

5. How would the cancer type influence your preference for receiving the results?

6. If you have a positive cancer result, how would you prefer for your family members to be involved?

7. Was your health screening examination today required by your company?

7a. Do you wish for your results to be sent to your company?

7b. Can you explain why you feel that way?

7c. Would the screening test result, that is, a test negative for cancer or positive for cancer influence your preference for involving your company?

8. Has anyone in your family had cancer before?

8a. Who was involved?

8 b. What happened?

8c. How has that influenced your thinking about truth telling of the cancer diagnosis?

9. Is there anything else you think is important for me to know about cancer screening tests or disclosure of the cancer diagnosis?

\section{ACKNOWLEDGMENTS}

This work was previously presented at the annual meeting of the North American Primary Care Research Group, Orlando, Florida, 1997. This investigation and Dr. Fetters' participation was made possible by the generous support of the University of Michigan Center for Japanese Studies; the Japanese Business Society of Detroit Foundation; and the Lyle C. Roll Program for $\mathrm{Hu}-$ mane Medical Practice. A grant from the Japan Foundation for Aging and Health supported Dr. Masuda's participation. The authors also acknowledge all the patients who participated and generously shared their time, attitudes, values, experiences, and beliefs with the authors.

\section{REFERENCES}

1. Kleinman A, Eisenberg L, Good B: Culture, illness, and care: Clinical lessons from anthropological and cross cultural research. Ann Intern Med 1978;88: 251-258.

2. Kleinman A, Kunstadter P, Alexander ER, Russell G, James L: Culture and Healing in Asian Societies: Anthropological, Psychiatric, and Public Health Studies. Boston: G.K. Hall, 1978, pp. 1-462.

3. Kleinman A: Patients and Healers in the Context of $\mathrm{Cul}$ ture: An Exploration of the Borderland Between Anthropology, Medicine, and Psychiatry. Berkeley: University of California Press, 1980, pp. 1-427.

4. Stein HF: American Medicine as Culture. Boulder, $\mathrm{CO}$ : Westview Press, Inc., 1990, pp. 1-281.

5. Helman CG: Culture, Health, and Illness: An Introduction for Health Professionals. 3rd ed. Oxford, Boston: Butterworth-Heinemann Ltd, 1994, pp. 1-446.

6. Freund PES, McGuire MB: Health, Illness and the Social Body: A Critical Sociology. Englewood Cliffs, NJ: Prentice Hall, 1995, pp. 1-402.

7. Payer L: Medicine and Culture: Varieties of Treatment in the United States, England, West Germany, and France. New York: Henry Holt and Company, Inc, 1996, pp. 1-204.

8. Flanagan L: Managing diversity: The mandate of the 90's. Fam Pract Manage, 1996:31-38.

9. Kreier R: Crossing the cultural divide. Am Med News 1999;(Jan 25):12-14.

10. Kim H: Managing diversity. Am Med News 1999;(Jan 25):39-40, 42-43.

11. Culturally Effective Communication. American Medical News 1999;Feb 22:34.

12. Holland JC, Geary N, Marchini A, Tross S: An international survey of physician attitudes and practice in regard to revealing the diagnosis of cancer. Cancer Invest 1987;5:151-154.

13. Surbone A: Truth telling to the patient. IAMA 1992;268:1661-1662.

14. Estape J, Palombo H, Hernandez E, Daniels M, Estape T, Grau JJ, Vinolas N, Mane JM: Cancer diagnosis disclosure in a Spanish hospital. Ann Oncol 1992;3:451454.

15. Thomsen OO, Wulff HR, Martin A, Singer A: What do gastroenterologists in Europe tell cancer patients? Lancet 1993;341:473-476.

16. Pronzato P, Bertelli G, Losardo P, Landucci M: What 
do advanced cancer patients know of their disease? A report from Italy. Supp Care Cancer 1994;2:242-244.

17. Harrison A, Al-Saadi AH, Al-Kaabi AO, Al-Kaabi RS, Al-Bedwawi SM, Al-Kaabi OM, Al-Neaimi SBS: Should doctors inform terminally ill patients? The opinions of nationals and doctors in the United Arab Emirates. I Med Ethics 1997;23:101-107.

18. Surbone A, Zwitter M: Communication with The Cancer Patient-Information and Truth. New York: New York Academy of Sciences, 1997.

19. Long SO, Long BD: Curable cancers and fatal ulcers, attitudes toward cancer in Japan. Soc Sci Med 1982; 16:2101-2108.

20. Shibazaki T: Group wants doctors to inform patients more fully. Japan Times International Edition July 15-21, 1991:7.

21. Chang Y: Cancer patients speak out. Japan Times, Oct. 22, 1992, p. 17.

22. Kurtenbach E: Horrors haunt Japan's vaunted medical system. Japan Times, May 8, 1993.

23. Murakami A: Level with terminal patients, let them plan, surgeon urges. Japan Times, May 9, 1993:3.

24. Kristof ND: Tokyo journal-When doctor won't tell cancer patient the truth. New York Times February 24, 1995:4.

25. Murakami T: Religion of the patient and medical ethics. Kango Tenbo 1982;7:687-691.

26. Tanida N: Patients' rights in Japan. Lancet 1991;337: 242-243.

27. Swinbanks D: Japanese doctors keep quiet. Nature 1989:339:409.

28. Swinbanks D: When Silence isn't golden. Nature 1992;356:368.

29. Hadfield P: Tokyo perspective: Informed consent. Lancet 1993;34:1141.

30. Elwyn TS, Fetters MD, Gorenflo DW, Tsuda T: Cancer disclosure in Japan: Historical comparisons, current practices. Soc Sci Med 1998;46:1151-1163.

31. Fetters MD: The family in medical decision making: Japanese Perspectives. I Clin Ethics 1998;9:132-146.

32. Ministry of Health and Welfare: FY 1994 report on the socioeconomic survey of vital statistics: Terminal care [in Japanese]. Statistics and Information Department, Minister's Secretariat, 1994.

33. Morioka Y: Informed consent and truth telling to cancer patients. Gastroenterol Japonica 1991;26:789792.

34. Anonymous: (Gan to songenshi ni kansuru zenkoku seron chosa). [A national public opinion survey on cancer and living wills] Tokyo Yomiuri Chokan, July 7, 1993, p. 2.

35. Anonymous: Gan kokuchi "shirasenai" ga $58 \%$, sakunen yori 4 pointo zou [Cancer disclosure (to a family member) "Don't disclose" $-58 \%$, a four point increase over last year]. Tokyo Yomiuri Chokan, July 19, 1994, p. 2.

36. Higuchi N: The patient's right to know of a cancer diagnosis: A comparison of Japanese paternalism and American self-determination. Washburn Law 1992; 31:455-473.

37. Emanuel L, Emanuel E: The medical directive: A com- prehensive advance care document. JAMA 1989;261: 3288-3293.

38. Orentlicher D: From the Office of the General Counsel: Advance medical directives. IAMA 1990;263: 2365-2367.

39. Emanuel L, Barry M, Stoeckle J, Ettelson LM, Emanuel EJ: Advance directives for medical care: $A$ case for greater use. N Engl J Med 1991;324:889-895.

40. Asai A: Should physicians tell patients the truth? West J Med 1995;163:36-39.

41. Asai A, Kishino M, Fukui T, Sakai M, Yokota $M$, Nakata K, Sasakabe S, Sawada K, Kaiji F: A report from Japan. Choices of Japanese patients in the face of disagreement. Bioethics 1998;12:162-172.

42. Freedman B: Offering truth: One ethical approach to the uniformed cancer patient. Arch Intern Med 1993;153:572-576.

43. Crabtree BF, Miller WL: A qualitative approach to primary care research: The long interview. Fam Med 1991;23:145-151.

44. Ventres WB, Frankel RM: Ethnography: A stepwise approach for primary care researchers. Fam Med 1996;28:52-56.

45. Olesen V: Feminisms and models of qualitative research. In: Denzin NK, Lincoln YS, (eds): Handbook of Qualitative Research. Thousand Oaks: Sage Publications, Inc, 1994, pp. 158-174.

46. Creswell JA: Data collection. Qualitative Inquiry and Research Design. Choosing Among Five Traditions. Thousand Oaks: Sage Publications, Inc, 1998, pp. 109-137.

47. Waitzkin $\mathrm{H}$ : On studying the discourse of medical encounters: A critique of quantitative and qualitative methods and a proposal for reasonable compromise. Med Care 1990;28:473-488.

48. Bernard HR: Field notes: How to take, code, and manage them. In: Research Methods in Anthropology: Qualitative and Quantitative Approaches. 2nd ed. Walnut Creek, CA: AltaMira Press, 1995, pp. 180-207.

49. Morse JM: Designing funded qualitative research. In: Denzin NK, Lincoln YS, (eds): Handbook of Qualitative Research. Thousand Oaks: Sage Publications, Inc, 1994, pp. 220-235.

50. Creswell JA: Five qualitative traditions of inquiry. Qualitative Inquiry and Research Design. Choosing Among Five Traditions: Sage Publications, Inc., 1998, pp. 47-72.

51. Miller WL, Crabtree BF: Primary care research: a multimethod typology and qualitative road map. In: Crabtree BF, Miller WL, (eds): Doing Qualitative Research. Newbury Park: Sage Publications, 1992, pp. 3-28.

52. Lind S, Kopans D, Good, M. Patients' preferences for learning the results of mammographic examinations. Breast Cancer Res Treat 1992;23:223-232.

53. Matsumura S, Fukuhara S, Bitou S, et al: An examination of wishes of Japanese concerning cancer disclosure and the various factors influencing it. Nihon Iji Shinpo 1997;3830:37-42.

54. Oga H, Horie K, Yafune H, Sata N, Sata F: Shoukakikei shinkougan no byoumei to byouki no 
kokuchi-Daigaku byouin gekai no shinri ni oyobosu yogo no eikyou [Truth telling of disease name and stage in advanced gastrointestinal cancer-The psychologial effect of prognosis on surgeons at university hospital] Jap J Cancer Clin 1997;43:1477-1483.

55. Fetters MD, Danis M: We live too short and die too long-On Japanese and U.S. physicians' caregiving practices and approaches to withholding life sustaining treatments. In: Long SO, (ed): Caring for the Elderly in Japan and the US: Practices and Policies. New York: Routledge, 2000, pp. 121-145.

56. Lynn J, Teno J: A care provider perspective on advance directives and surrogate decision making for incompetent adults in the United States. In: Sass $\mathrm{H}-\mathrm{M}$, Veatch RM, Kimura R, (eds): Advance Directives and Surrogate Decision Making in Health Care-United States, Germany, and Japan. Baltimore: The Johns Hopkins University Press, 1998, pp. 3-33.

57. La puma J, Orentlicher D, Moss R: Advance directives on admission: Clinical implications and anlysis of the Patient Self-Determination Act of 1990. JAMA;1991: 402-405.

58. Schonwetter R, Walker R, Kramer D, Robinson BE: Resuscitation decision making in the elderly: The value of outcome data. I Gen Intern Med 1993;8:295300.

59. Hanson LC, Rodgman E: The use of living wills at the end of life. Arch Intern Med 1996;156:1080-1082.

60. Landry F, Kroenke K, Lucas C, Reeder J: Increasing the use of advance directives in medical outpatients. J Gen Intern Med 1997;12:412-15.

61. Gross M: What do patients express at their preferences in advance directives? Arch Intern Med 1998;158:363-365.

62. Blackhall L, Murphy S, Frank G, et al: Ethnicity and attitudes towards patient autonomy. JAMA 1995;274: 820-825.

63. Schneider CE: The Practice of Autonomy-Patients, Doctors, and Medical Decisions. New York: Oxford University Press, 1998.

64. Katz J: The Silent World of Doctor and Patient. New York: The Free Press, Division of Macmillan, Inc., 1984, pp. 1-263.

65. Veatch R: Death, Dying, and the Biological Revolution: Our last quest for responsibility. New Haven, CT: Yale University Press, 1989.

Address reprint requests to: Michael D. Fetters, M.D., M.P.H., M.A. Japanese Family Health Program Department of Family Medicine University of Michigan Health Systems 1018 Fuller Street Ann Arbor, MI 48109-0708

E-mail: mfetters@umich.edu 
This article has been cited by:

1. Joshua S. Barclay, Leslie J. Blackhall , James A. Tulsky . 2007. Communication Strategies and Cultural Issues in the Delivery of Bad NewsCommunication Strategies and Cultural Issues in the Delivery of Bad News. Journal of Palliative Medicine 10:4, 958-977. [Abstract] [PDF] [PDF Plus]

2. Shinji Matsumura, Seiji Bito, Honghu Liu, Katharine Kahn, Shunichi Fukuhara, Marjorie Kagawa-Singer, Neil Wenger. 2002. Acculturation of Attitudes Toward End-of-life Care. A Cross-cultural Survey of Japanese Americans and Japanese. Journal of General Internal Medicine 17:7, 531-539. [CrossRef] 